\title{
NOTE ON THE THEOREM OF GENERALIZED FOURIER'S CONSTANTS.
}

BY PROFESSOR W. D. A. WESTFALL.

IN the theory of the development of arbitrary functions $f(x)$ in series of normalized orthogonal functions $\psi_{i}(x)$,

$$
\begin{gathered}
f(x)=\sum_{1}^{\infty} a_{i} \psi_{i}(x), \\
a_{i}=\int_{a}^{b} f(x) \psi_{i}(x) d x, \quad \int_{a}^{b} \psi_{i}(x) \psi_{k}(x) d x \begin{cases}=0, & i \neq k, \\
=1, & i=k,\end{cases}
\end{gathered}
$$

sufficient conditions that this equality exists, and that the series converges uniformly, are in general that $f(x)$ and its first $m-1$ derivatives are continuous in $(a, b)$ and satisfy homogeneous boundary conditions for $x=a$ and $x=b{ }^{*}$ Then there follows immediately the fundamental theorem of "generalized" Fourier's constants

$$
\int_{a}^{b}\{f(x)\}^{2} d x=\sum_{1}^{\infty} a_{i}^{2}
$$

This note will give a simple proof that, in case (1) holds true for every function satisfying the above conditions, it holds true for every integrable function $f(x)$, such that $\{f(x)\}^{2}$ is integrable. $\dagger$

Since $f(x)$ and $\{f(x)\}^{2}$ are integrable in $(a, b)$ there exists, for every $\epsilon>0$, a division of $(a, b)$ in a finite number of subintervals $\left(x_{1}, x_{2}\right),\left(x_{2}, x_{3}\right), \cdots,\left(x_{n-1}, x_{n}\right), x_{1}=a, x_{n}=b$, such that a function $\phi(x)$ can be defined, having the following properties :

$$
|\phi(x)|=\text { lower bound of } f(x) \text { in }\left(x_{i}, x_{i+1}\right) \text { for } x_{i} \leqq x<x_{i+1},
$$

$$
\begin{gathered}
\phi(x) f(x) \geqq 0, \\
\left|\int_{a}^{b}\left[\{f(x)\}^{2}-\{\phi(x)\}^{2}\right] d x\right|<\frac{\epsilon}{2} .
\end{gathered}
$$

* D. Hilbert, "Zweite Mitteilung über Integralgleichungen," Göttinger Nachrichten, 1904.

E. Schmidt, Dissertation, Göttingen, 1905.

$\uparrow$ The theorem has been proven essentially by W. Stekloff with the restriction that $f(x)$ be bounded, Mémoires de l'Académie de St. Pétersbourg, 1904. The above proof is simpler and does away with this restriction. 
Consider now a function $F(x)$ defined as follows : in the $\delta$-neighborhood of $x_{i}$,

$$
\begin{gathered}
F(x)=\phi\left(x_{i}+\delta\right) \frac{\int_{x_{i}}^{x}\left(x-x_{i}\right)^{m}\left(x-\delta-x_{i}\right)^{m} d x}{\int_{x_{i}}^{x_{i}+\delta}\left(x-x_{i}\right)^{m}\left(x-\delta-x_{i}\right)^{m} d x},\left(x_{i} \leqq x \leqq x_{i}+\delta\right), \\
F(x)=\phi\left(x_{i}-\delta\right) \frac{\int_{x_{i}}^{x}\left(x-x_{i}\right)^{m}\left(x+\delta-x_{i}\right)^{m} d x}{\int_{x_{i}}^{x_{i}-\delta}\left(x-x_{i}\right)^{m}\left(x+\delta-x_{i}\right)^{m} d x},\left(x_{i}-\delta \leqq x \leqq x_{i}\right),
\end{gathered}
$$

where $0<\delta<$ minimum $\frac{1}{2}\left(x_{k}-x_{k-1}\right),(i, k=1,2, \ldots, n)$. For other values of $x, F(x)=\phi(x) . \quad F(x)$ and its first $m-1$ derivatives are continuous and vanish for $x=a=x_{1}$ and $x=b=x_{n}$. Moreover,

$$
|F(x)| \leqq|\phi(x)|, \quad F(x) \phi(x) \geqq 0 .
$$

\section{Hence}

$$
\begin{aligned}
\left|\int_{a}^{b}\{\phi(x)\}^{2}-\{F(x)\}^{2} d x\right| \leqq \int_{x_{1}}^{x_{1}+\delta} \phi^{2}(x) d x & +\int_{x_{n}-\delta}^{x_{n}} \phi^{2}(x) d x \\
& +\sum_{2}^{n-1} \int_{x_{i}-\delta}^{x_{i}+\delta} \phi^{2}(x) d x .
\end{aligned}
$$

Since $\phi(x)$ is bounded, $\delta$ can be chosen so small that

$$
\int_{a}^{b}\{\phi(x)\}^{2}-\{F(x)\}^{2} d x \leqq \frac{1}{2} \epsilon .
$$

From (2), (3), (4)

$$
\begin{gathered}
|F(x)| \leqq|f(x)|, \quad F(x) f(x) \geqq 0, \\
\left|\int_{a}^{b}\{f(x)\}^{2}-\{F(x)\}^{2} d x\right|<\epsilon, \\
\{f(x)-F(x)\}^{2} \leqq\{f(x)\}^{2}-\{F(x)\}^{2} .
\end{gathered}
$$

From a known theorem there exists the following inequality for any set of continuous normalized orthogonal functions $\psi_{i}(x)$ and any integrable function $f(x)$ such that $|f(x)|$ is also integrable : 


$$
\int_{a}^{b}\{f(x)\}^{2} d x \geqq \sum_{1}^{\infty} a_{i}^{2}, \quad a_{i}=\int_{a}^{b} f(x) \psi_{i}(x) d x .
$$

Hence the identity exists

$$
\begin{gathered}
\int_{a}^{b}\{f(x)\}^{2} d x-\sum_{1}^{\infty} a_{i}^{2}=\int_{a}^{b}\left[\{f(x)\}^{2}-\left\{F^{\prime}(x)\right\}^{2}\right] d x \\
\quad+\int_{a}^{b}\{F(x)\}^{2} d x-\sum_{1}^{\infty} A_{i}^{2}+\sum_{1}^{\infty}\left(A_{i}-a_{i}\right)\left(A_{i}+a_{i}\right) . \\
a_{i}=\int_{a}^{b} f(x) \psi_{i}(x) d x, \quad A_{i}=\int_{a}^{b} F(x) \psi_{i}(x) d x
\end{gathered}
$$

Moreover

$$
\left|\sum_{1}^{\infty}\left(A_{i}-a_{i}\right)\left(A_{i}+a_{i}\right)\right| \leqq \sqrt{\sum_{1}^{\infty}\left(A_{i}-a_{i}\right)^{2} \cdot \sum_{1}^{\infty}\left(A_{i}+a_{i}\right)^{2}}
$$

or from (6)

$$
\begin{gathered}
\leqq \sqrt{\int_{a}^{b}\{F(x)-f(x)\}^{2} d x \cdot \int_{a}^{b}\{F(x)+f(x)\}^{2} d x} \\
<\sqrt{\epsilon \int_{a}^{b}\{2 f(x)\}^{2} d x} \leqq 2 \sqrt{\epsilon \int_{a}^{b}\{f(x)\}^{2} d x}
\end{gathered}
$$

since $|F(x)| \leqq|f(x)|$ and $F(x) f(x) \geqq 0$.

Applying this inequality with (5) in (7),

$$
\begin{aligned}
\left|\int_{a}^{b}\{f(x)\}^{2} d x-\sum_{1}^{\infty} a_{i}^{2}\right| \leqq \epsilon+\int_{a}^{b}\{ & F(x)\}^{2} d x-\sum_{1}^{\infty} A_{i}^{2} d x \\
& +2 \sqrt{\epsilon \int_{a}^{b}\{f(x)\}^{2} d x .}
\end{aligned}
$$

This inequality holds for any $\epsilon>0$ and the corresponding function $F(x)$. Hence the theorem :

If for a set of normalized orthogonal functions $\psi(x)$ and every function $f(x)$, which with its first $m-1$ derivatives is continuous in $(a, b)$ and satisfies for $x=a, x=b$ a set of homogeneous boundary conditions, there exists the equality

$$
\int_{a}^{b}\{f(x)\}^{2} d x=\sum_{1}^{\infty}\left\{\int_{a}^{b} f(x) \psi_{i}(x) d x\right\}^{2},
$$


then this equality holds true for every integrable function $f(x)$ such that $\{f(x)\}^{2}$ is integrable.

GöTTINGEN,

July 17, 1908.

\section{ON THE LOGICAL BASIS OF GRASSMANN'S EXTENSIVE ALGEBRA.}

BY MR. A, R. SCHWEITZER.

\section{$\S 1$.}

Is studying the algebra of Grassmann fundamentally, we must carefully distinguish between the Ausdehnungslehre proper and the Ausdehnungslehre in a broad sense. Grassmann himself makes no rigid separation of the two viewpoints ; generally, however, the former is found in the edition of 1844 and the latter is in the edition of 1862 and in various memoirs.* Briefly, we may say that the Ausdehnungslehre proper for $n$ dimensions $(n=1,2,3, \ldots)$ is a development of $n$-dimensional euclidean geometry by means of the outer product of $n+1$ points, which fundamentally is reducible to sameness of sense of two $(n+1)$-hedra. It consists of descriptive axioms and certain axioms which relate exclusively to $n$-spatial congruence. On the basis of these axioms and their consequences, we arrive at the broader conception of the Ausdehnungslehre by means of suitable abstraction, the introduction of parameters, "formalization," etc. $\dagger$

\section{$\S 2$.}

If we take three dimensions, the fundamental properties of the Ausdehnungslehre are as follows. Concretely expressed, the basal relation is sameness of sense of two tetrahedra (identical or not) which is implied by what Grassmann has called "Gleichbezeichnung." $\$$ This relation is fundamentally nonmetrical, and solely in terms of it we may construct a system of postulates for three-dimensional descriptive geometry which is

\footnotetext{
* Cf. the Collected Works of Grassmann. For references to the Ausdehnungslehre and related subjects we may refer to Macfarlane's admirable bibliography, Dublin, 1904.

† For instance, see Crelle, vol. 49, p. 123 ; Math. Annalen, vol. 12, p. 376 ; Ausdehnungslehre, 1862, $z \&$ 151-215; Study, Wiener Berichte, vol. 91, p. 111. $\ddagger$ Coll. Works, I, p. 303, 304.
} 